\title{
Binding and Encapsulation of Doxorubicin on Smart Pectin Hydrogels for Oral Delivery
}

\author{
Valeria E. Bosio • Victoria Machain • \\ Azucena Gómez López • Ignacio O. Pérez De Berti • \\ Sergio G. Marchetti • Magdalena Mechetti • \\ Guillermo R. Castro
}

Received: 6 November 2011 / Accepted: 23 February 2012 /

Published online: 14 March 2012

(C) Springer Science+Business Media, LLC 2012

\begin{abstract}
Pectins (Pec) of 33 to $74 \%$ esterification degree were tested with doxorubicin (Dox), a very high toxic drug widely used in cancer therapies. Pec with 35 and $55 \% \mathrm{DE}$ were selected because of the Dox binding higher than Pec microspheres of 35 and $55 \%$ obtained by ionotropic gelation with $\mathrm{Ca}^{+2}$ have 88 and $66 \%$ Dox loading capacity. Kinetic Dox release showed more than 80.0 and about $30.0 \%$ free drug from $35 \%$ and $55 \% \mathrm{Pec}$ formulations at $\mathrm{pH} 7.4$, and $37{ }^{\circ} \mathrm{C}$ after 1 -h incubation, respectively. Besides, Dox release decrease to $12 \%$ in $55 \%$ Pec microsphere formulation after 1 -year storage at $4{ }^{\circ} \mathrm{C}$. FTIR analysis of Pec-Dox complex showed hipsochromic shifts for the $\sigma_{\mathrm{C}=\mathrm{O}}, \delta_{\mathrm{N}-\mathrm{H}}$ and $\sigma_{\mathrm{C}-\mathrm{C}}$ vibrational modes compared to Dox spectrum suggesting strong interaction between the drug cargo and the matrix. Rheological studies of Pec and Pec-Dox samples flow behavior exhibited a shear-thinning nature. Fifty-five percent of Pec showed higher viscosity than the viscosity for $35 \%$ Pec in all range of temperatures analyzed, and decreased when the temperature is raised. Besides, Pec-Dox complexes have higher viscosity values than those of the corresponding Pec samples, and viscosity curves as function of shear rate for $35 \%$ Pec-Dox are above the curves of $55 \%$ Pec-Dox. In both cases, the results are confirming significant interaction between the cargo and the matrix, which also was established in viscoelastic dynamic analysis.
\end{abstract}

Keywords Drug delivery $\cdot$ Doxorubicin $\cdot$ Pectin $\cdot$ FTIR $\cdot$ Rheology

\footnotetext{
V. E. Bosio • V. Machain · G. R. Castro $(\bowtie)$

CINDEFI (Institute of Applied Biotechnology, UNLP - CONICET CCT La Plata), School of Sciences, Universidad Nacional de La Plata, Calle 50 \# 227, 1900 La Plata, Argentina

e-mail: grcastro@gmail.com
}

\section{A. G. López • M. Mechetti}

Lab. de Física de Fluídos y Electroreología, Depto. de Física, Facultad de Ciencias Exactas y Tecnología, Universidad Nacional de Tucumán, Av. Roca 1800, T4001MVB Tucumán, Argentina

I. O. P. De Berti - S. G. Marchetti

CINDECA (CONICET, CCT La Plata), Universidad Nacional de La Plata, Calle 47 \# 257, La Plata 1900, Argentina 


\section{Introduction}

New trends in the pharmaceutical technologies are involving biomaterials in drug formulations because of their remarkable properties such as high chemical structure diversity, stereospecific functionalities, biological properties, wide span of molecular weights, and mostly lack of toxic side effects [1]. Particularly, mild synthesis and biodegradability, plus convenient rheological and thickening properties, make biopolymers very attractive molecules useful for a myriad of potential applications. Polymer-based particles synthesis can be summarized in four basic mechanisms: covalent and ionotropic crosslinking, polyelectrolyte complex, and self-assembly of modified polysaccharides [2, 3].

Drug encapsulation offers many advantages, such as: (1) the protection of the drug against in vivo degradation, (2) the reduction of potential toxic side effects associated with the drug doses, (3) the increase in patient comfort by avoiding repetitive bolus injection or the use of perfusion pumps, (4) more favorable drug pharmacokinetics, and (5) optional design of drug sustainable release. The main difficulties arise in controlling the molecule release, while also to find a compromise between encapsulation efficiency, drug leakage induced by diffusion and/or by matrix degradation, the biocompatibility and biodegradation of particle components and the formulation processes [4-8]. Drug formulations depend on the choice of suitable carrier showing high encapsulation efficiency, improved bioavailability, desired target, retention time, and environmental factors among other factors. Among them, rheological properties influence each step of the pharmaceutical processing, such as filling, mixing, and gelling. Also, rheological properties (e.g., viscosity) of the matrix affect the formulation behavior in the digestive tract, and the profile release of loaded drugs $[4,9$, 10]. However, desired micro- and nano-medicines are generally formulated by hit and trial method but without rationale $[11,12]$.

Doxorubicin hydrochloride (Dox) is used in the treatment of many solid tumors, such as breast, lung, stomach, or ovarian cancer and sarcoma. Dox inhibits cell growth by DNA intercalation and inhibition of topoisomerase II among other action over cell metabolism. Unfortunately, Dox is highly toxic, by biodistribution to non-targeted tissues causing severe side effects, which are limiting the anthracycline dosage and use. At least two Dox commercial formulations were in the market: liposomal Doxil ${ }^{\circledR}$ and albumin-based Abraxane ${ }^{\circledR}$, but other formulations are still on different trial phases and reviewed recently [13]. However, many studies are still carried out with Dox, giving rise to various Doxcontaining micro-nanoparticles, such as polymeric carriers, inorganic magnetic nanoparticles where Dox is adsorbed onto their surface, or even solid lipid nanoparticles containing Dox as an ion-pair complex [13]. Recent trends in Dox delivery involve the development of environmentally sensitive matrices showing dependence of $\mathrm{pH}$, temperature, or ions [1416]. Since Dox is a hydrophilic and hydrosoluble molecule, the main challenge is still entrapping Dox with a significant encapsulation rate and yield into such micro- and/or nanoparticulate systems with high shelf-life stability, and establish kinetic release. Therefore, new formulation strategies via Dox encapsulated in particulate drug delivery systems are still required to be developed. Among them, oral delivery has the advantages of being noninvasive, simple to be handled, no specialist required, and special places to be administered which is particularly relevant in inmuno-suppressed patients and IV administration impose the risks of secondary infections.

Natural biopolymers are promising candidates for the development of sustainable drug delivery systems [17]. Particularly, food-grade biopolymers like alginate, pectin, guar gum carrageenin has numerous advantages such us they are cheap, biodegradable, renewable, easy to handle, non-toxic, and commercially available in large quantities. Additionally, some 
food-grade biopolymers are native environmentally sensitive molecules implying that nonchemical modification and purification is required for pharmaceutical purposes.

The aim of the present work is to examine the interaction of selected biopolymers with Dox as cargo molecule model. Twelve biopolymers as potential matrices for Dox cargo were screened, and the best system was selected to develop a hydrogel microsphere carrier, as a typical model for delivery of aromatic anticancer drugs. Rheological studies and FTIR analysis were used to characterize the matrix. Pec gel microspheres were selected and optimized based on loading capacity and controlled release kinetic. The kinetic analysis was developed under in vitro physiological conditions. Studies of the microspheres were complemented by optical and fluorescence microscopy.

\section{Materials and Methods}

Chemicals

Biopolymers used in this work were: low viscosity sodium alginate (Alg) (average $\mathrm{M \eta} 1 \times 10^{5} \mathrm{Da}$ ) obtained from Biochem (USA); gelatine (Gel) from Parafarm (Argentina); arabic gum (Ag) from Droan (Argentina); guar gum (GG) from Nutrial (Argentina); garrofin gum (GarG) and carrageenin (Carr) from Saporiti (Argentina); pectins (Pec) with 33.0, 35.4, 55.3, and 67.2 \% esterification degree (ED), from CPKelco (Argentina), pectin $74 \% \mathrm{ED}$ (Pec 74) from Sigma-Aldrich (USA). Doxorubicin (Dox) was a gift from LMK pharmaceuticals (Argentina). Carboxy Methyl Guar Gum (CMGG) was kindly provided by Prof. Arup Mukherjee (University of Calcutta, India). All other reagents used were of analytical grade purchased from Sigma-Aldrich (St. Louis, MO, USA) or Merck (Darmstadt, Germany).

Dox was quantified by spectrofluorimetry (Perkin Elmer LS 50B, Japan) using $\lambda_{\text {exc }} 454 \mathrm{~nm}$ and $\lambda_{\mathrm{em}} 588 \mathrm{~nm}$ with appropriate calibration curve [18].

Preparation of Biopolymer Aqueous Solutions

Polymer concentrations between $0.5 \%$ and $2.0 \%$, were made in MiliQ water quality (Millipore, USA) supplemented with $10 \mu \mathrm{M}$ sodium azide and kept at $5{ }^{\circ} \mathrm{C}$ up to use (see Table 1).

Determination of the Interaction Dox-Biopolymers

Different 0.5 to $2.0 \%$ biopolymer solutions $(375 \mu \mathrm{l})$ were mixed $155 \mu \mathrm{M}$ Dox solution $(125 \mu \mathrm{l})$ for $15 \mathrm{~min}$ and $2 \mathrm{~h}$ at $25^{\circ} \mathrm{C}$ (Table 1). Later, $1.0 \mathrm{ml}$ of child acetone or ethanol was

Table 1 Biopolymers used in the binding test of Dox

\begin{tabular}{lc}
\hline Biopolymers & Concentration $(\% p / v)$ \\
\hline GEL & 2.0 \\
ALG & 1.5 \\
PEC & 1.5 \\
CARR & 1.0 \\
GUM & 0.5 \\
\hline
\end{tabular}


added to precipitate the biopolymer-cargo molecule complex, and then centrifuged at $10,000 \times g$ for $5 \mathrm{~min}$. Dox concentration was measured in supernatant.

For pectins, $2.0 \%$ biopolymer containing $300 \mu \mathrm{M}$ Dox in $50 \mathrm{mM}$ MOPS buffer $(\mathrm{pH}=7.0)$ was incubated at $25{ }^{\circ} \mathrm{C}$ under magnetic agitation $(500 \mathrm{rpm})$ for $2 \mathrm{~h}$.

Biopolymer Formulations, Microsphere Formation

Pectin hydrogel microspheres from $33.0 \%$ to $67.2 \%$ DE were tested to determine optimal conditions for microsphere stability and Dox entrapment.

Pectins were dissolved in $50 \mathrm{mM}$ MOPS buffer $(\mathrm{pH}=7.0)$ with agitation and mixed with or not a volume of Dox solution.

Hydrogel microspheres were prepared by extrusion through a $100-\mu \mathrm{m}$ syringe attached to a pump (Watson-Marlow, UK). Pec solutions with or without Dox $(300 \mu \mathrm{M})$ were dropped into a solution of $100 \mathrm{mM}$ Tris- $\mathrm{HCl}$ buffer $(\mathrm{pH}=8.9)$ containing $50 \mathrm{mM} \mathrm{CaCl}_{2}$ at $0{ }^{\circ} \mathrm{C}$ under continuous stirring $(200 \mathrm{rpm})$ for $20 \mathrm{~min}$. Microspheres were aged in calcium chloride solution for $1 \mathrm{~h}$, followed by filtration on paper (Whatman no. 1). Dox concentration was assayed in the supernatants. Filtered microspheres were kept in solution containing $50 \mathrm{mM}$ $\mathrm{CaCl}_{2}$ and $10 \mu \mathrm{M} \mathrm{aN}_{3}$ at $5{ }^{\circ} \mathrm{C}$ until the assays. Alternatively, microspheres were acetone-airdried at room temperature over night and then stored as mentioned before.

\section{Dox Loading}

Dox entrapment efficiency was determined by dissolving $100 \mathrm{mg}$ of microspheres in $5.0 \mathrm{ml}$ containing $100 \mathrm{mM}$ sodium phosphate buffer $(\mathrm{pH}=7.4)$, and centrifuged at $10,000 \times \mathrm{g}$ for 10 min $\left(5^{\circ} \mathrm{C}\right)$. Dox was spectrofluorometrically assayed in the supernatant as previously mentioned. The loading efficiency was evaluated as follows:

$$
\text { Entrapment efficiency }=\frac{\text { Dox concentration in the supernatant }}{\text { Theoretical Dox concentration }}
$$

Optical and Fluorescence Microscopy

Optical and fluorescence microscopies were carried out with an UV source leaned and filters (495-505 nm) for fluorescence observation respectively (Leica DM 2500, Germany).

\section{Rheological Measurements}

Controlled-stress rheometer (Anton Paar MCR 301) with a 43-mm parallel plate (PP43/GLSN16497 measurements system) and a P-PTD120-SN80470786 accessory system was used to characterize the rheological properties of Pec solutions. The gap used was $0.7 \mathrm{~mm}$. For steady shear measurements, shear rates ranging from 1 to $1,000 \mathrm{~s}^{-1}$ were used and the resulting stress was recorded. All rheological measurements were carried out at $37{ }^{\circ} \mathrm{C}$ and the reported curves were mean values of four measurements. Shear stress, shear rate, and apparent viscosity data are obtained directly from the instrument.

Vibrationals Analysis: FTIR Spectroscopies

Pec and Pec-Dox formulations were analyzed by FTIR (JASCO FT/IR - 4200). Pellets were prepared by mixing the samples at $5.0 \% w / w$ with potassium bromide $(\mathrm{KBr}$, Pike 
technologies, UK) and scanned with background correction at 256 number scans, against a high-energy ceramic source and DLATGS detector. FTIR scans corresponding to Pec and Pec-Dox were recorded in the 4,000-400 $\mathrm{cm}^{-1}$ with $4-\mathrm{cm}^{-1}$ resolution. Also, Dox formulations were analyzed by FTIR at the SMIS beamline (Soleil, French National Synchrotron Facility, France).

\section{Dox Kinetic Release Studies from Pectin Microparticles}

Dehydrated spheres, $200.0 \mathrm{mg}$, were weighted in an analytical scale (Adventurer OHAUS) of high precision $(0.1 \mathrm{mg})$ and were placed in a Reacti-road flask of $4 \mathrm{ml}$ of capacity, with $1.0 \mathrm{ml}$ of buffer solution. The kinetic of Dox's release was ran at $37{ }^{\circ} \mathrm{C}$ and at $500 \mathrm{rpm}$. To certain intervals, samples of $300 \mu$ were withdraw and replaced with an equal volume of fresh buffer. Dox concentration was determined by fluorescence.

\section{Storage Effect in Dox Stability and Release}

Dox kinetic release experiments were performed in microparticles shelf-preserved at $4{ }^{\circ} \mathrm{C}$ for 6 months.

\section{Statistics}

Two or three independent experiments were conducted with a minimum of duplicates $(N=2)$ for each data point. In the case of pectins screening assay, four independent experiments were conducted with duplicates $(N=2)$ for each data point. Data for these measurements were analyzed using a Student's $t$ test. Statistically significant values were defined as $p<0.05$. Data in the graphs represent the mean \pm standard deviation.

\section{Results and Discussion}

\section{Screening for Biopolymer-Dox Binding}

Nine biopolymers with different chemical structure were challenged to Dox. Only three biopolymers presented binding with Dox after 15-min incubation (data not shown), but the number increases to four biopolymers with Dox after 2-h incubation: two pectins of $33.0 \%$ and $74 \%$ ED displayed high interaction, Carr showed intermediate and Alg a low interaction with the Dox molecule (Fig. 1). The Dox binding of 37.9, 39.0, and 46.8, $49.0 \%$ were found for 33 and 74 Pecs during 15 and 120 min, respectively. For Carr and Alg, the Dox binding were 20.0, 0.0 and $24.6,20.0 \%$ in 15 and $120 \mathrm{~min}$, respectively. The differences in the binding among the polymer-Dox interaction within the incubation time are suggesting a kinetic-driven process. After $15 \mathrm{~min}$, the binding process probably is still in a transient equilibrium, where the interaction is more energetically that kinetically favorable in our experimental conditions.

Pectins were selected for further binding studies, and the interaction between Pecs with different ED and Dox was screened (Fig. 2). However, the results of the interaction between Pec and Dox were not obvious visually, and also under Student $t$ test, no significant differences at $95 \%$ confidence were found. 
Fig. 1 Precipitation of Dox-biopolymer complexes after 2-h incubation

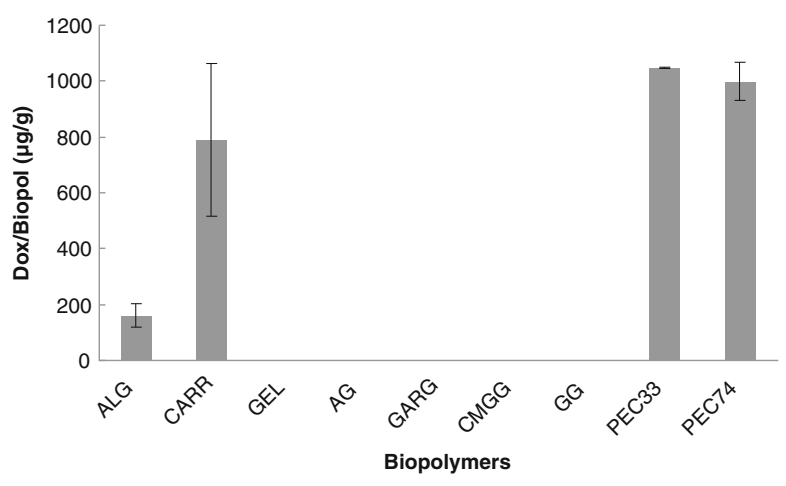

Gelation Conditions and Release Kinetic Studies

Ionotropic gelation of pectins showed Dox encapsulation higher than $50 \%$ for Pecs with 35 and higher ED (data not shown). Particularly, 35 and 55 Pecs showed 88 and $66 \%$ Dox encapsulation, respectively.

In order to maximize the Dox encapsulation in the Pec matrixes, buffers at different pHs and water-miscible organic solvents were used in the gelling solution. However, no significant differences were observed in all systems tested (data not shown). These results are indicating that the ionization Dox molecule is not relevant for the gel encapsulation or entrapment. In the presence of organic solvents, Pec gelation rate is faster rather than in aqueous medium. Nevertheless, no differences on Dox encapsulation in water and organic solvents were found. These results are suggesting that Dox encapsulation in the tested pectins is not related to the Pec gelation process, meaning Dox physical entrapment, but probably related to some interaction between the drug and the matrix.

On the other side, gelation of Pecs with different ED showed significant differences of Dox encapsulation. Pecs with low ED showed better gelation capacity in presence of $\mathrm{Ca}^{+2}$ compared to high ED Pecs. Additionally, pectin microspheres with low and medium ED displayed more resistance to mechanical to shear stress compared to highly methylated ones (data not shown). However, the effect of methyl groups (ED) in the biopolymer could be relevant in the Pec-Dox interaction since they can be associated to hydrophobic properties of pectins, by making hydrophilic/hydrophobic clusters in the Pec molecule. Consequently, Pec hydrophobic patches could increase the interaction with the Dox aromatic ring.

Based on the encapsulation results, 35 and 55 Pecs were selected for further formulation studies.

Fig. 2 Effect of pectin methylation degree in Doxpectin complex formation

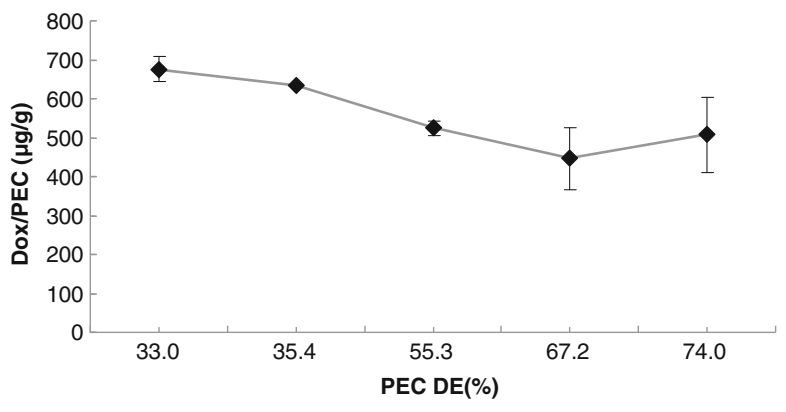


Fig. 3 Flow behavior of pectin and pectindoxorubicin complex. Shear stress as a function of shear rate. Symbols: $35 \%$ Pec (white square); $35 \%$ Pec-doxorubicin (black square); $55 \%$ Pec (white diamond); $55 \%$ Pecdoxorubicin (black diamond)

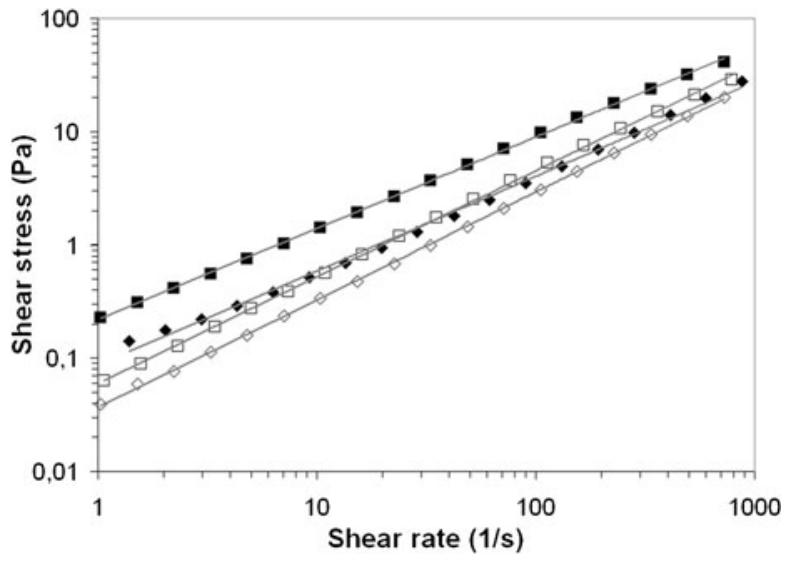

Rheological Studies

The apparent viscosity (pascal seconds, $\mathrm{Pa} \mathrm{s}$ ), shear stress (pascal) and shear rate (per second) at different rpm were recorded. The shear rate was increased between 0 and $1,000 \mathrm{~s}^{-1}$ within a period of $175 \mathrm{~s}$ for recording the "up" curve then it was reduced from $1,000-0 \mathrm{~s}^{-1}$ for recording the "down curve" in the same period of time to study the timedependent nature of the samples. A slight tixotropy (the area between the curves was negligible) was detected only for 35 and $55 \%$ Pec-Dox. For these samples, the down curve was used. Natural log curves of shear stress against shear rate have been presented in Fig. 3.

Apparent viscosity was found to decrease with an increase in shear rate indicating a shear thinning behavior. The apparent viscosity was greater for the low-grade pectin and was found to increase to increase almost half decade for the systems containing doxorubicin. For $55 \mathrm{Pec}$, less resistance to shear flow is observed. These observations were reinforced by the results obtained from dynamic viscoelastic properties of the samples, not shown here. That Pecs with increasing ED which exhibited lower viscosity were in agreement with similar observations reported previously $[9,10]$. The apparent viscosity at $1 \mathrm{~s}^{-1}$ was approximately 0.06 and $0.04 \mathrm{~Pa}$ s for 35 and $55 \mathrm{Pecs}$, respectively, but those values grows up to $0.22 \mathrm{~Pa}$ s for 35 Pec-Dox and 0.102 Pa s for 55 Pec-Dox and has been presented in Fig. 4.

Fig. 4 Flow behavior of pectin and pectin-doxorubicin complex. Apparent viscosity as a function of shear rate. Symbols: $35 \%$ Pec (white square); $35 \%$ (black square); $55 \% \mathrm{Pec}$ (white diamond); $55 \%$ Pec-doxorubicin (black diamond)

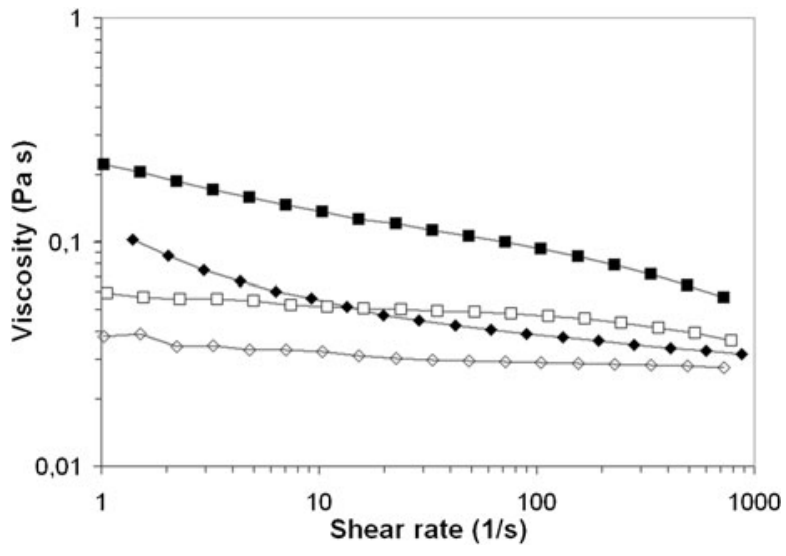


Table 2 Rheological characteristics of pectins with and without doxorubicin

\begin{tabular}{lccr}
\hline Samples & Flow behavior index & Consistency index $K(\mathrm{~Pa} \mathrm{~s})$ & $R^{2}$ \\
\hline Pec 35\% & 0.939 & 0.060 & 0.9997 \\
Pec 55\% & 0.952 & 0.037 & 0.9998 \\
Pec 35 \%+ doxorubicin & 0.807 & 0.218 & 0.9997 \\
Pec 55 \%+ doxorubicin & 0.833 & 0.087 & 0.9972 \\
\hline
\end{tabular}

\section{Rheology Model}

The shear stress-shear rate data obtained during experimentation was fitted to the power law model to determine the consistency coefficient $(K)$ and flow index $(n)$. The constitutive equation of this model is $\tau=K(\gamma)^{n}$, where $\tau$ is the shear stress (pascal), $\gamma$ the rate of shear (per second), $K$ the consistency index (pascal per second ${ }^{n}$ ) and $n$ the flow behavior index which is a measure of the degree of the non-Newtonian behavior. For shear-thinning fluids, the flow index $n$ lies between zero and unity with values further removed from unity indicating a more pronounced non-Newtonian behavior.

Least square regression analysis was applied in log form and the consistency index, the flow behavior index, and the correlation coefficient $(R)$ were evaluated. They have been reported in Table 2. As can be seen the value of $n$ is less than 1. Thus, the studied substances were found to exhibit shear-thinning behavior. The Pec-Dox samples showed more pronounced shear-thinning characteristics as can be observed in Fig. 4.

From the rheological analysis, $55 \mathrm{Pec}$ was selected as matrix for Dox encapsulation.

Spectroscopies Studies: FTIR Analysis

FTIR analysis confirmed the presence of an interaction between the $55 \mathrm{Pec}$ and Dox (Table 3). Characteristic Dox N-H group peak shifted to a higher value from 1,615 to $1,628 \mathrm{~cm}^{-1}\left(\Delta \nu=13 \mathrm{~cm}^{-1}\right)$. Also, the $1,722 \mathrm{~cm}^{-1}$ Dox carbonyl group peak shifted to $1,733 \mathrm{~cm}^{-1}\left(\Delta v=11 \mathrm{~cm}^{-1}\right)$.

Table 3 Major FTIR peaks assignments for Dox molecule, 55 \% HM pectin, and formulation Dox/HM pectin at $\mathrm{pH}=7.4$

\begin{tabular}{lllll}
\hline Wavenumbers $\left(\mathrm{cm}^{-1}\right)$ & & $\Delta v\left(\mathrm{~cm}^{-1}\right)$ & Assignments \\
\cline { 1 - 2 } Dox & HM pectin & Formulation & & \\
\hline 3,389 & - & 3,380 & & $\delta(\mathrm{N}-\mathrm{H})$ \\
3,330 & - & 3,269 & & $\delta(\mathrm{H}-\mathrm{O})$ \\
2,932 & 2,936 & 2,929 & & $\sigma(\mathrm{C}-\mathrm{H})$ \\
1,722 & 1,743 & 1,733 & $11-10$ & $\sigma(\mathrm{C}-\mathrm{C}), v(-\mathrm{COOCH})$ \\
1,615 & 1,609 & 1,628 & $13-19$ & $\delta(\mathrm{N}-\mathrm{H}), \gamma(-\mathrm{COOH}), v(\phi=\mathrm{O})$ and phenyl \\
& & & & breathing modes \\
1,408 & - & $1,459,1,443$ & $\sim 43$ & $\sigma(\mathrm{C}-\mathrm{C})$ \\
- & 1,328 & 1,331 & & $\sigma(\mathrm{C}-\mathrm{O}-\mathrm{C})$ \\
1,077 & 1,100 & 1,099 & & $\sigma(\mathrm{C}-\mathrm{O})$ \\
\hline
\end{tabular}


In addition, the carboxyl group of $55 \mathrm{Pec}$ shifted from 1,609 to $1,628 \mathrm{~cm}^{-1}\left(\Delta \nu=19 \mathrm{~cm}^{-1}\right)$. Bands associated to the aromatic Dox motifs shifted from 1,743 to $1,733 \mathrm{~cm}^{-1}\left(\Delta \nu=10 \mathrm{~cm}^{-1}\right)$, suggesting a possibly interaction made by the pectin hydrophobic pockets (highly methylated carboxylates) with the hydrophobic motif of anthracycline: the aromatic ring (Table 3).

Pec-Dox complex showed too an hipsochromic shifts for the $\sigma_{\mathrm{C}-\mathrm{C}}$ vibrational mode compared to Dox spectrum.

FTIR studies are strongly suggesting a complex interaction among the cargo and the matrix (Table 3) [19-21].
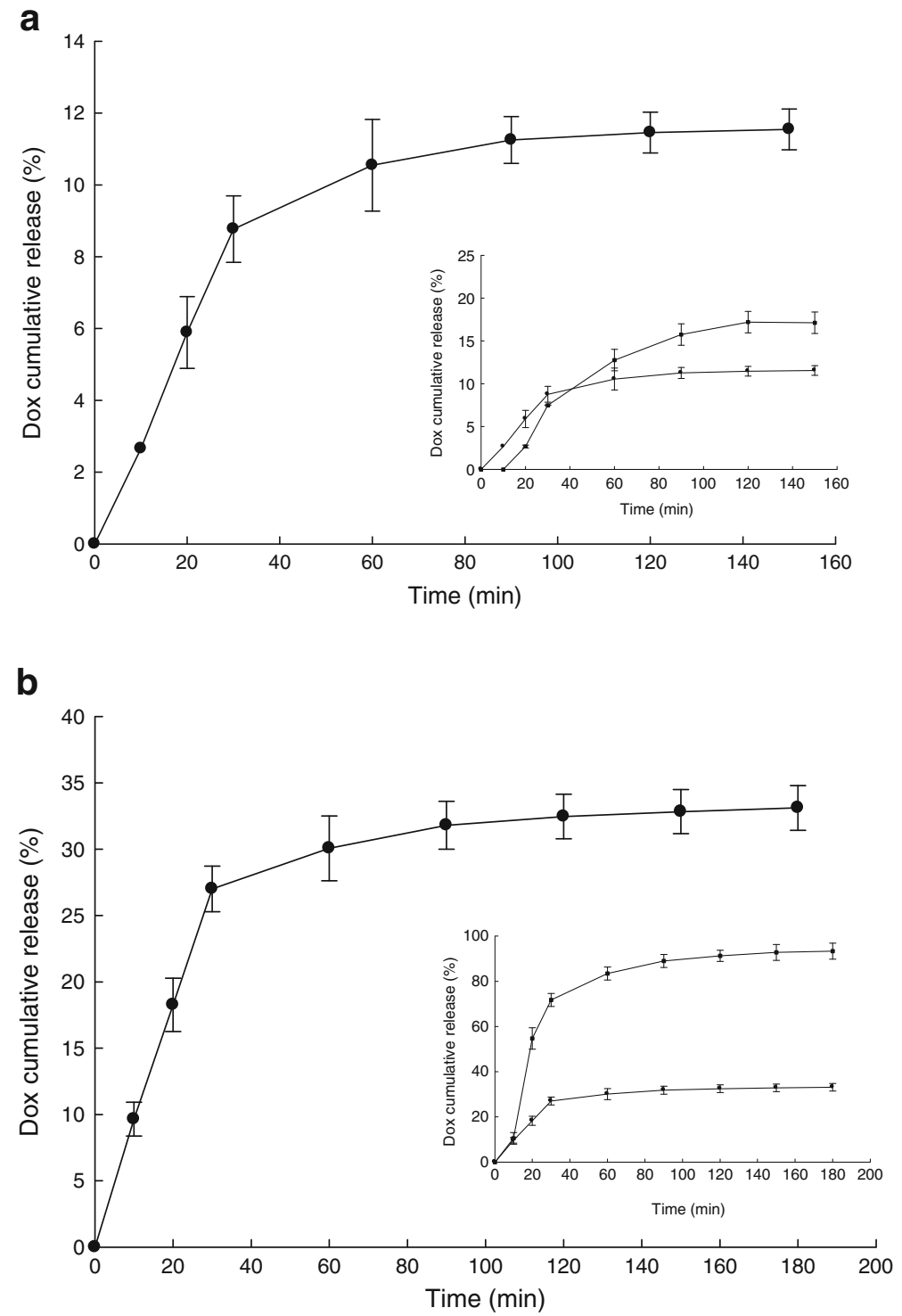

Fig. 5 Dox kinetic release profiles from $35 \%$ and $55 \%$ Pec gel microspheres at pH 2.0 (a) and 7.4 (b) and $37^{\circ} \mathrm{C}$, respectively. Symbols: $35 \%$ Pec (white square) and $55 \%$ Pec (black circle) 
Dox Release Studies

Pecs of 35 and $55 \%$ ED released approximately 17.0 and $12.0 \%$ Dox, respectively, after 2-h incubation in buffer at $\mathrm{pH} 2.0$ and $37{ }^{\circ} \mathrm{C}$ (Fig. 5a). Later, Dox release plateau was reached (Fig. 5), suggesting that Dox is mostly associated to the external layers in the $35 \mathrm{Pec}$ microspheres. This fact can be explained by the high interaction between calcium ions and pectin chains making high cross-linked gel, in consequence the Dox molecule could be extruded from the inner microsphere layers. On the contrary, $55 \%$ Pec showed lower amount of Dox release in $2 \mathrm{~h}$, and because of high ED (20\%) the ionic interaction of the pectin molecule chain with $\mathrm{Ca}^{+2}$ is less strong and the incidence of hydrophobic group potentially is higher than from the $35 \%$ Pec.

Also, the results shown in Fig. 5 are indicating that both 35 and $55 \%$ Pecs microspheres are stable at least for $2 \mathrm{~h}$ under our experimental conditions under acidic conditions ( $\mathrm{pH} 2.0$ ).

Under intestinal $\mathrm{pH}(\approx 7.4)$, the differences on Dox release from $35 \%$ to $55 \%$ ED Pecs are more drastics: more than $80 \%$ Dox release from microspheres were observed in $35 \% \mathrm{ED} \mathrm{Pec}$, meanwhile about $30 \%$ Dox release were observed on $55 \%$ ED Pec. Also, $55 \%$ ED Pec matrix formulation showed very stable and constant Dox release values lower than $35 \%$ at least in $3 \mathrm{~h}$ (Fig. 5b).

\section{Storage Conditions and Dox Loading}

Dox release from $55 \%$ Pec gel microspheres stored for 6 months at $4{ }^{\circ} \mathrm{C}$ was $12 \%$ in $3 \mathrm{~h}$ under the same experimental acid conditions. The results are indicating that the gel microsphere structure was preserved along this period of time, and the positive effect of biogel aging on Dox release (Fig. 6).

\section{Conclusions}

In the present work, Dox encapsulation in pectin gels was found correlated to the inverse relationship between calcium concentration and pectin esterification degree. Pectins with intermediate ED (35 and $55 \%$ ) showed high Dox encapsulation inversely proportional to the ED. On the other side, Dox release from $35 \% \mathrm{Pec}$ is about 2.5 times faster compared to than in $55 \% \mathrm{Pec}$ at $\mathrm{pH} 7.4$ suggesting an effect of carboxylate esterification on drug release. The
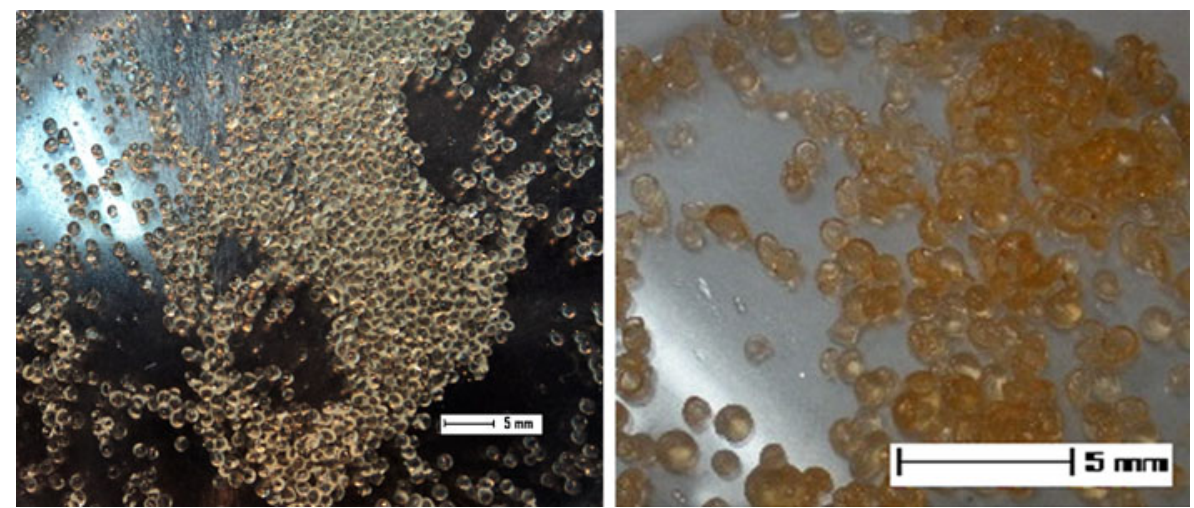

Fig. 6 Optical microscopy for $55 \%$ PEC particles with and without Dox (left and right, respectively) 
hydrophobic interaction of $55 \%$ Pec with Dox should be higher than $35 \%$ Pec, considering $20 \%$ of ED difference. Also, the high ED could impact on microsphere structure reducing the gel stability, but no differences on gel microsphere stability in both types of microspheres in the time frame tested were observed. A compromise between ionotropic gelation (calcium concentration) and esterification degree of pectins should be considered in order to obtain microspheres with high loading capabilities but also good gel stability. Fifty-five percent Pec showed $66 \%$ Dox encapsulation and excellent stability after 6 month storage. These results allowed to considered oral administration of Dox as an alternative in colon chemotherapies.

Vibrational spectroscopy and rheological analysis of Pec-Dox complex were significant since they allowed to establish type of interactions and fluid behavior which is relevant in order to understand the interactions between the cargo (Dox) and the matrix (55\% Pec), and determine the behavior of the formulation under physiological environments.

Based on the results presented in the present work, $55 \%$ Pec biogels seems to be an excellent candidate for the development of Dox encapsulation in gel matrix. Studies on matrix aging conditions, new hybrid materials using the Pec-Dox system, effect of Dox-Pec formulation on mammalian cell cultures are under way in our laboratories.

Acknowledgments The present work was supported by Consejo Nacional de Investigaciones Científicas y Técnicas (CONICET), and Agencia Nacional de Promoción Científica y Técnica (ANPCyT) of Argentina. We thank Dr. Paul Dumas (SMIS beam line, Soleil Synchrotron Facility, France) for his kind support and expertise during the sample analysis. We thank Mrs. Graciela Guananja from CPKelco (Buenos Aires, Argentina) for her kind help and the donation of pectin samples; and to Dr. Mario Malaspina (Laboratorio LKM S.A.) and Dr. Nicolas Martinez from Quality Pharma Laboratories for the doxorubicin samples used in the present work.

\section{References}

1. Williams, D. F. (2009). Biomaterials, 30, 5897-5909.

2. Liu, Z., Jiao, Y., Wang, Y., Ziyong, C., \& Zhang, D. (2008). Advanced Drug Reviews, 60, 1650-1662.

3. Dornish, M., Kaplan, D., \& Skaugrud, O. (2001). Annual New York Academy of Sciences, 944, 388-397.

4. Chi, H., Moturi, V., \& Lee, Y. (2009). Journal of Controlled Release, 136, 88-98.

5. Peng, C., Zhao, Q., \& Gao, C. (2010). Colloids and Surfaces A: Physicochemical and Engineering Aspects, 353, 132-139.

6. Nielsen, O. S., Reichardt, P., Christensen, T. B., Pink, D., Daugaard, S., Hermans, C., Marreaud, S., van Glabbeke, M., Blaye, J., \& Judson, I. (2006). European Journal of Cancer, 42, 2303-2309.

7. Vrignaud, S., Anton, N., Gayet, P., Benoit, J. P., \& Saulnier, P. (2010). European Journal of Pharmaceutics and Biopharmaceutics, 79, 197-204.

8. von Maltzahn, G., Park, J. H., Lin, K. Y., Singh, N., Schwöppe, C., Mesters, R., Berdel, W. E., Ruoslahti, E., Sailor, M. J., \& Bhatia, S. N. (2011). Nature Materials, 10, 545-552.

9. Bockki, M., Jongbin, L., Sanghoon, K., Kwang-Geun, L., SungHo, L., \& Suyong, L. (2011). Bioresource Technology, 102, 3855-3860.

10. Fraeye, I., Doungla, E., Duvetter, T., Moldenaers, P., Van Loey, A., \& Hendrickx, M. (2009). Food Hydrocolloids, 23, 2069-2077.

11. Kumari, A., Yadav, S. K., \& Yadav, S. C. (2010). Colloids and Surfaces. B, Biointerfaces, 75, 1-18.

12. Liechty, W. B., Kryscio, D. R., Slaughter, B. V., \& Peppas, N. A. (2010). Annual Review of Chemical and Biomolecular Engineering, 1, 149-173.

13. Malam, Y., Loizidou, M., \& Seifalian, A. M. (2009). Trends in Pharmacological Sciences, 30, $592-599$.

14. Seo, S., Lee, C.-S., Jung, Y.-S., \& Na, K. (2012). Thermo-sensitivity and triggered drug release of polysaccharide nanogels derived from pullulan-g-poly(l-lactide) copolymers. Carbohydrate Polymers, 87, 1105-1111.

15. Manchun, S., Dass, C. R., \& Sriamornsak, P. (2012). Targeted therapy for cancer using pH-responsive nanocarrier systems. Life Sciences. doi:10.1016/j.lfs.2012.01.008. 
16. Cai, H., Caihua Ni, C., \& Zhang, L. (2012). Preparation of complex nano-particles based on alginic acid/ poly[(2-dimethylamino) ethyl methacrylate] and a drug vehicle for doxorubicin release controlled by ionic strength. European Journal of Pharmaceutical Sciences, 45, 43-49.

17. Oh, J. K., Lee, D. I., \& Park, J. M. (2009). Biopolymer-based microgels/nanogels for drug delivery applications. Progress in Polymer Science, 34, 1261-1282.

18. Karukstisu, K. K., Thompson, E. H. Z., Whiles, J. A., \& Rosenfeld, R. J. (1998). Biophysical Chemistry, 73, 249-263.

19. Kayal, S., \& Ramanujan, R. V. (2010). Materials Science and Engineering: C, 30, 484-490.

20. Purcell, J. M., \& Fishman, M. L. (1987). Carbohydrate Research, 159, 185-190.

21. Lin-Vien, D., Colthup, N. B., Fateley, W. G., \& Grasselli, J. G. (1991). The handbook of infrared and Raman characteristic frequencies of organic molecules. Boston: Academic. 